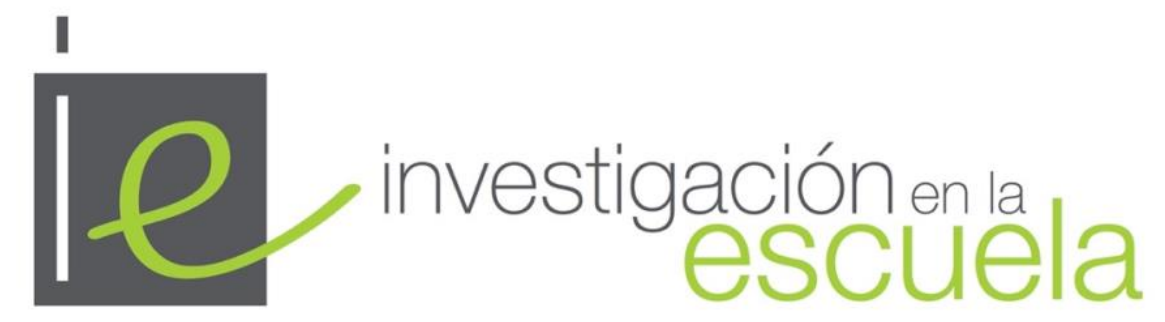

Revista de Investigación e Innovación Educativa nº 102, 2020 | e-ISSN 2443-9991

\title{
Emociones y percepciones sobre indagación de profesorado en formación inicial
}

\author{
Pre-service teachers' emotions and perceptions about inquiry
}

iD Cristina García-Ruiz es Investigadora Postdoctoral en la Facultad de Ciencias de la Educación de la Universidad de Málaga (España)·crisgarcia@uma.es·https://orcid.org/0000-0002-9623-7832

iD Teresa Lupión-Cobos es Profesora Contratada Doctora en la Facultad de Ciencias de la Educación de la Universidad de Málaga (España)·teluco@uma.es·https://orcid.org/0000-0002-6937-7178

iD Ángel Blanco-López es Catedrático en la Facultad de Ciencias de la Educación de la Universidad de Málaga (España)·ablancol@uma.es·https://orcid.org/0000-0003-3628-0801

Cómo citar este artículo

García-Ruiz, C., Lupión-Cobos, T. y Blanco-López, Á. (2020). Emociones y percepciones sobre indagación de profesorado en formación inicial. Investigación en la Escuela, 102, 54-70. doi: http://doi.org/10.12795/IE.2020.i102.04

Resumen. En los últimos años, la investigación didáctica sobre el perfil emocional del profesorado ha ganado especial relevancia, dada la repercusión que su diagnóstico y autorregulación tienen en el proceso de enseñanza. El uso de enfoques innovadores, como la indagación, lleva además asociado una serie de emociones que pueden derivar en dificultades para su aplicación, resultando por tanto esencial incorporar su estudio en los programas de formación inicial. Por ello, este trabajo analiza las posibles relaciones entre las emociones asociadas a los procesos de indagación, la percepción sobre su aprendizaje y la aplicabilidad en las clases de ciencia, que manifiestan ocho profesores de secundaria en formación inicial de la especialidad de Física y Química del Máster de Profesorado. Se ha utilizado un método mixto en el que se emplea como instrumentos un cuestionario y las reflexiones de los participantes tras realizar una actividad de indagación. Los resultados muestran una percepción favorable a la indagación, con una prevalencia de emociones positivas, así como una mejora en la percepción del aprendizaje sobre las etapas de indagación tras completar la actividad. En general, observamos una predisposición a implementar este tipo de actividades, aunque no encontramos relación entre la percepción sobre la aplicabilidad y las emociones, pero sí con la percepción sobre su propio aprendizaje.

Abstract. In recent years, educational research on the teachers' emotional profile has gained particular relevance, given the impact that its diagnosis and self-regulation has on the teaching process. The use of innovative approaches, such as inquiry, is also associated with a series of emotions that can lead to difficulties in their application, making it essential to incorporate their study into initial training programs. For this reason, this work describes the relationship between the emotions associated with the inquiry processes and the perception of their learning in a sample of eight pre-service secondary Physics and Chemistry teachers of the Master's Degree in Teaching. Through a mixed-method (quantitativequalitative) that uses as instruments a questionnaire and the reflections of the participants after carrying out an inquiry activity, we provide the data obtained around the emotions identified in the stages of the inquiry process, their relationship with learning and future implementation in the classroom. The results show a favourable perception of the inquiry, with a prevalence of positive emotions, as well as an improvement in the perception of learning about the inquiry stages after completing the activity. In general, we observed a predisposition to implement these types of activities, although we did not find any relationship between the perception of applicability and emotions, but with the perception of their learning. 
Palabras clave $\cdot$ Keywords

Emociones, percepciones, indagación, formación inicial, profesorado de ciencias, educación secundaria Emotions, perceptions, inquiry, pre-service teaching, science teachers, secondary education

\section{Introducción y objetivos}

En los últimos años, el interés de los estudiantes por la ciencia ha disminuido considerablemente (Organisation for Economic Co-operation and Development [OECD], 2017), lo que demanda un cambio educativo hacia metodologías que promuevan un mayor interés y motivación hacia su aprendizaje, con dinámicas que brinden oportunidades para un desarrollo competencial, trabajando en equipo a través de experiencias de resolución de problemas abiertos. Dentro de las metodologías consideradas efectivas, la indagación, impulsada por el conocido como «Informe Rocard» (Rocard et al., 2007), ha emergido recientemente como un enfoque que puede ayudar a la transformación necesaria en la enseñanza de las ciencias, contribuyendo a la alfabetización científica de los estudiantes y al desarrollo de una educación inclusiva.

Este nuevo paradigma hacia un profesorado promotor de la enseñanza por indagación, precisa que los docentes se conviertan en facilitadores del aprendizaje, adoptando para ello modelos constructivistas (Crawford, 2014). Este proceso requiere de un tiempo prolongado debido a la dificultad para influenciar en las creencias y valores previos del profesorado, y a los cambios que se tienen que producir no sólo a nivel cognitivo o procedimental, sino también afectivo. Algunos estudios muestran cómo el profesorado de ciencias puede experimentar determinadas emociones durante su proceso formativo, repercutiendo éstas en su eficacia y confianza como futuros docentes (Yeigh et al., 2016).

A pesar de que estos factores ponen de manifiesto la necesidad de incorporar la enseñanza por indagación en los programas de formación inicial, frecuentemente el componente emocional queda relegado a un segundo plano (Mellado et al., 2014), pudiendo derivar en dificultades para incorporar cambios didácticos de carácter innovador. Por tanto, la comprensión e identificación de las emociones experimentadas por el profesorado durante la enseñanza por indagación, conociendo en qué momentos se inician y por qué, puede facilitar la propia regulación emocional, ayudándole a afrontar de forma positiva situaciones que provocan emociones negativas (Ritchie et al., 2013), y favorecer la adopción plena de este enfoque didáctico. Así, tal y como señalan Jiménez-Liso et al. (2019), resulta fundamental involucrar a los docentes en formación inicial en experiencias que, además de profundizar en la enseñanza por indagación, propicien oportunidades para el compromiso emocional.

Por ello, en este trabajo analizamos las emociones que experimentan los futuros docentes de ciencias de secundaria al realizar una actividad que les introducen en las etapas del ciclo de indagación (identificación del problema, formulación de hipótesis o comunicación de resultados, entre otras). También se abordan otras dos cuestiones relativas a las percepciones de este profesorado: su reflexión sobre su propio aprendizaje y sobre la aplicabilidad de la indagación en las clases de ciencias. Consideramos que las relaciones, si las hay, entre sus emociones y estas percepciones nos permitirán identificar aquellos aspectos de la indagación que potencialmente puedan suponer un reto para su futura práctica.

\section{Marco teórico}

\subsection{Emociones asociadas a los procesos de indagación}

Dentro de las emociones académicas (de logro, temáticas, sociales y epistémicas) (Bellocchi et al., 2017), son estas últimas, las epistémicas, que refieren al propio aprendizaje y se centran en el conocimiento o su construcción, las que resultan especialmente importantes durante el aprendizaje con tareas nuevas, manifestándose habitualmente en situaciones de información contradictoria o incongruente, cuando se cuestionan las representaciones cognitivas de los estudiantes o se logran nuevas comprensiones (Pekrun et al., 2018). Enfrentarse a situaciones novedosas o que originen un cambio conceptual a menudo involucra una respuesta emocional (Chiu et al., 2014). Además, el aprendizaje complejo con frecuencia provoca también emociones más negativas de confusión, aburrimiento y frustración (D’Mello y Graesser, 2012). Durante los procesos de indagación, las emociones, especialmente las epistémicas y las de logro aunque también en menor medida las temáticas (Palmer, 2009), cobran especial relevancia, al estar asociadas íntimamente al razonamiento en la práctica científica (Davis y Bellocchi, 2018). 
El aspecto emocional vinculado al enfoque de indagación como estrategia metodológica y su inclusión en la formación inicial, ha comenzado a explorarse recientemente, con algunos estudios llevados a cabo con profesorado de primaria (Alvarado et al., 2019; Sánchez-Martín et al., 2018), y de secundaria (Jiménez-Liso et al., 2019). En general, los resultados alcanzados por Alvarado et al. (2019) y Jiménez-Liso et al. (2019) son convergentes, describiendo ambos un dominio de las emociones positivas asociadas a la realización de actividades o secuencias de indagación en los cursos de formación. Esto demuestra el efecto favorecedor de aplicar este tipo de estrategias con el profesorado en formación inicial, ya que contribuyen a un conocimiento y autorregulación emocional en los docentes que ayuda a minimizar el posible rechazo que puedan experimentar hacia la puesta en práctica de este tipo de actividades, consecuencia de emociones negativas como la frustración, el aburrimiento o la ira (Tomas et al., 2016). En este sentido, ambos autores señalan la necesidad de hacer conscientes a los docentes, a través de la reflexión, de la conectividad entre su perfil emocional y su futura identidad docente.

Sendos estudios longitudinales, en los que se valoró la experiencias de los participantes antes y después de realizar la indagación, coinciden al destacar «interés» y «satisfacción» como las emociones positivas mayoritarias, afianzando el hecho de que la indagación se percibe como un enfoque motivador hacia el aprendizaje, actuando a su vez como un catalizador en las interacciones e intercambios emocionales (Alvarado et al., 2019).

Jiménez-Liso et al. (2019) además profundizan en las emociones negativas, y destacan la «inseguridad» en los momentos anteriores a la actividad, asociada a las etapas de formulación de hipótesis, justificación, análisis y predicción de los resultados, entre otras. En su trabajo constatan cómo a lo largo del tiempo esa emoción da paso a la «confianza» y «satisfacción», infiriendo por tanto un carácter no tan negativo en esa inseguridad que acompaña al desarrollo del nuevo aprendizaje.

Sánchez-Martin et al. (2018) analizan la respuesta emocional al abordar diferentes estrategias innovadoras, diferenciando, en su caso, la indagación (a la que se refiere como «investigación guiada») de las actividades prácticas de laboratorio tipo «receta», que muchos docentes suelen considerar como indagación (Constantinou et al., 2018). En ambas estrategias, se identifica un cierto porcentaje de «rechazo» (del 5\%), que además en el caso de la indagación, lleva asociado un menor porcentaje de emociones positivas, y que atribuyen al hecho de la complejidad que implica la resolución de problemas de investigación, aspecto ausente en una actividad de laboratorio de carácter meramente reproductivo.

\subsection{Percepciones del profesorado sobre la indagación}

A pesar de las numerosas publicaciones que avalan las ventajas del enfoque de indagación (Chen et al., 2014; Marshall et al., 2016), su práctica docente supone todavía un reto, como consecuencia de una amplia gama de mitos, creencias y experiencias previas (Vázquez y Manassero, 2015) que repercuten en el propio concepto que tiene el profesorado de la indagación.

Su falta de implementación en el aula no deja de ser llamativa, considerando el énfasis que ha recibido la enseñanza por indagación en las reformas educativas de las últimas décadas (Sjøberg, 2019). Todavía muchos docentes consideran que, aunque puede ser una buena estrategia, la indagación no prepara a los estudiantes para los siguientes niveles de escolarización, no ajustándose a la realidad de la enseñanza. Asimismo, en los pocos casos en los que se aplica este enfoque, la conceptualización errónea de la enseñanza por indagación y la naturaleza de la ciencia culmina en una pobre implementación (McLaughlin y MacFadden, 2014).

En la transición desde estudiante de ciencias a profesorado en formación inicial, a menudo se contemplan percepciones ligadas al conocimiento del contenido pedagógico (Kang et al., 2013). Sin embargo, es habitual encontrar una desconexión con los procesos asociados a la enseñanza de la ciencia por indagación, que especialmente en el ámbito de la formación inicial del profesorado de secundaria, demanda una exposición y práctica explícitas para tener éxito en su transferencia al aula (Bartley, 2019).

En lo referente al proceso de instrucción, varios estudios apuntan a la influencia de la percepción sobre el rol docente durante la indagación, asociado a una falta de confianza a nivel de habilidades docentes, que frecuentemente se traduce en indagaciones con un alto grado de guía de las actividades implementadas (Krämer et al., 2015; Nuangchalerm, 2017; Qablan, 2008). También se resaltan otros factores identificados como dificultades en el uso de este enfoque, como los procesos de planificación y evaluación, la gestión del aula o los recursos y restricciones propios del sistema educativo (Abril et al., 2014; Romero-Ariza et al., 2020).

Un factor importante a considerar es la existencia de relaciones significativas entre las creencias epistemológicas y aspectos propios de la naturaleza de la ciencia o la meta-cognición, ampliamente analizadas por diferentes autores (Crawford, 2014; Zion et al., 2018), que recomiendan la reflexión explícita durante las 
experiencias de indagación, trabajando específicamente aquellas percepciones más difíciles de modificar. Consecuentemente, la realización de propuestas que brinden experiencias prácticas de indagación enfocadas a la comprensión del proceso proporciona oportunidades para mejorar la capacidad docente del profesorado en formación inicial de cara a guiar a su futuro alumnado en este enfoque de enseñanza-aprendizaje.

\section{Preguntas de investigación}

Este estudio se plantea obtener respuestas con respecto a las siguientes preguntas:

1. ¿Qué emociones manifiestan profesores de Física y Química en formación inicial cuando llevan a cabo una actividad de indagación?

2. ¿Qué percepciones tienen sobre su propio aprendizaje después de realizar la actividad de indagación?

3. ¿Qué percepción tienen, después de realizar la actividad, sobre la aplicabilidad de la indagación en las clases de Física y Química?

4. ¿Qué relaciones, si las hay, aparecen entre las emociones manifestadas, la percepción de su aprendizaje y la aplicabilidad de la indagación?

\section{Metodología}

En este estudio se ha utilizado un método mixto (Bryman, 2006), a través de la combinación de enfoques cuantitativos y cualitativos, con la finalidad de obtener una imagen completa respecto a las emociones y percepciones del profesorado de secundaria en formación inicial (PSFI) sobre el enfoque de indagación. Concretamente, hemos empleado métodos cuantitativos para estudiar las tendencias en cuanto al perfil emocional y la percepción del aprendizaje, y métodos cualitativos para explorar las reflexiones de los PSFI durante el proceso.

Así, se llevó a cabo un proceso de triangulación que ocurrió en dos momentos diferentes: en el primero, se procedió al análisis global de todos los instrumentos por separado, fusionándolos en el segundo momento, de análisis individual. Se han vinculado las emociones experimentadas en cada una de las etapas de la indagación, con su percepción del aprendizaje al respecto y con las reflexiones que aportaron sobre su intencionalidad para llevar este tipo de actividades a su práctica docente.

\subsection{Participantes}

La investigación se ha realizado con ocho profesores de secundaria en formación inicial (PSFI) del Máster de Profesorado de la Universidad de Málaga, que cursaron la especialidad de Física y Química durante el curso 2019/2020, cuya caracterización se recoge en la tabla 1.

Tabla 1

Caracterización de la muestra de estudio

\begin{tabular}{cccccc}
\hline PSFI & Género & Edad & Titulación & $\begin{array}{c}\text { Experiencia } \\
\text { investigadora }\end{array}$ & $\begin{array}{c}\text { Experiencia } \\
\text { docente }\end{array}$ \\
\hline 1 & Masculino & Mayor de 30 & Química & No & Sí (clases particulares) \\
2 & Masculino & Mayor de 30 & Química & No & Sí (clases particulares) \\
3 & Femenino & Entre 25 y 30 & Física & Sí (doctorado) & Sí (clases particulares) \\
4 & Femenino & Mayor de 30 & Ingeniería Química & No & Sí (clases particulares) \\
5 & Masculino & Mayor de 30 & Ciencias Ambientales & No & No \\
6 & Masculino & Menor de 25 & Ciencias Ambientales & No & No \\
7 & Masculino & Mayor de 30 & Ingeniería Industrial & No & Sí (docencia \\
8 & Femenino & Mayor de 30 & Ciencias Ambientales & No & Sí (clases particulares) \\
\hline
\end{tabular}




\subsection{Instrumentos de toma de datos y método de análisis}

Los datos se registraron tras completar una actividad de indagación (adaptada de Muñoz-Campos et al., 2020), realizada como parte de un programa de formación específico en este enfoque (García-Ruiz et al., 2020). La actividad, centrada en los procesos de fermentación, involucraba a los PSFI a través de una indagación guiada en el proceso de preparación de yogur, trabajando colaborativamente. Así, tras plantear como pregunta inicial ¿cómo podemos preparar yogur?, los PSFI se involucraron, desde su rol como estudiantes, en las etapas del proceso indagatorio, planteando preguntas investigables, formulando hipótesis, seleccionando variables, realizando el diseño experimental y la recogido de datos, analizando los resultados y, finalmente, comunicando las conclusiones obtenidas y las nuevas preguntas derivadas de la indagación realizada.

A través de dos instrumentos (figura 1), un cuestionario (que incluía un apartado de selección múltiple de emociones y otro de valoración sobre la percepción del aprendizaje antes y después de realizar la actividad) y las reflexiones de los PSFI sobre el análisis didáctico de la actividad realizada, se pudieron obtener datos sobre sus emociones y percepciones acerca de la actividad de indagación realizada.

\section{Figura 1}

Instrumentos de investigación y esquema de toma de datos

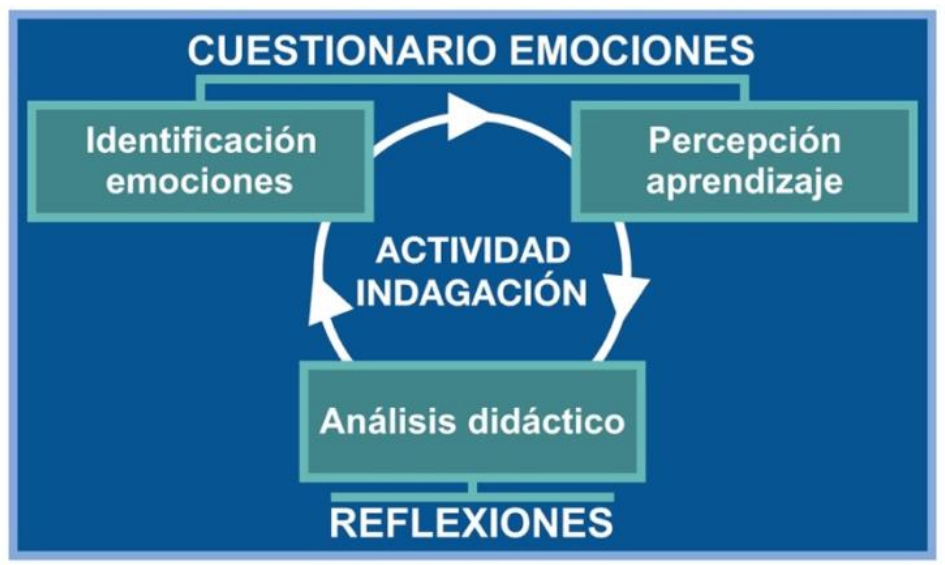

El cuestionario propuesto (tabla 2), basado en el trabajo de Jiménez-Liso et al. (2019), relacionaba las emociones asociadas a los procesos de indagación, incluyendo para ello emociones de logro (confianza, satisfacción, vergüenza e insatisfacción) y epistémicas (interés, concentración, aburrimiento, rechazo e inseguridad). Inicialmente se registraron las emociones seleccionadas por cada uno de los PSFI asociadas a las etapas del proceso de indagación (A-H) descritas en el trabajo de Ferrés-Gurt et al., (2015). Para su análisis, se realizó un estudio descriptivo cuantitativo con el programa RStudio (versión 1.3.1093), recogiendo las frecuencias de emociones y su representación porcentual.

\section{Tabla 2}

Cuestionario de emociones

\begin{tabular}{|c|c|c|c|c|c|c|c|c|c|}
\hline \multirow[b]{2}{*}{$\begin{array}{l}\text { Conocimiento sobre el } \\
\text { proceso de indagación }\end{array}$} & \multicolumn{9}{|c|}{ Emociones } \\
\hline & 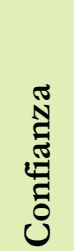 & 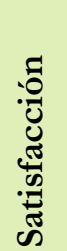 & 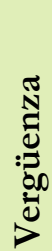 & 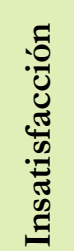 & & 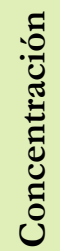 & 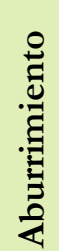 & 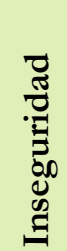 & 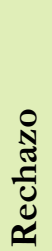 \\
\hline
\end{tabular}
A. Identificación de problemas investigables
B. Formulación de hipótesis
C. Identificación de variables
D. Planificación de la investigación 
E. Recogida y procesamiento de datos

F. Análisis de datos y obtención de conclusiones

G. Comunicación de resultados

H. Metarreflexión

A continuación, se preguntó a los PSFI, antes y después de realizar la actividad de indagación, por la percepción de su propio aprendizaje (Jiménez-Liso et al., 2019) en cada una de las etapas de la indagación, mediante una escala Likert de cinco puntos (1, no sé nada; 2, sé un poco; 3, lo sé bien; 4, lo sé muy bien; 5, puedo explicarlo a un amigo/a). Posteriormente se llevó a cabo un análisis cuantitativo de las puntuaciones obtenidas, utilizando nuevamente el programa RStudio, y analizando los cambios entre la percepción de aprendizaje antes y después.

Finalmente, se registraron las reflexiones de cada uno de los participantes, que respondían a las preguntas planteadas en la tabla 3, y cuyo análisis cualitativo se realizó inductivamente mediante técnicas de codificación realizadas con el software Atlas.ti (versión 8.4.4) (www.atlatsti.com/) (Saldaña, 2009).

Tabla 3

Preguntas directoras de la reflexión sobre la actividad de indagación

P1 ¿Qué ventajas encuentras en el tipo de actividad planteada en las sesiones anteriores?

P2 ¿Qué inconvenientes?

P3 ¿La aplicarías en tu clase? Justifica tu respuesta

P4 ¿Qué cambiarías y por qué?

Los investigadores leyeron varias veces los textos elaborados por los PSFI e identificaron aspectos de indagación relacionados con las preguntas establecidas en la tarea entregada. Un total de 34 códigos fueron utilizados, agrupados en cuatro categorías principales (ventajas, inconvenientes, aplicación y modificaciones). Este análisis se completó con una búsqueda por palabra clave para analizar las posibles menciones a las emociones consideradas o no en los cuestionarios.

\section{Análisis y resultados}

\subsection{Emociones sobre la indagación}

Los resultados globales obtenidos en el cuestionario de emociones aparecen en la tabla 4, en la que se recogen las frecuencias de cada una de las emociones por etapas y su ponderación de representación en el proceso. En ella aparecen relacionadas las etapas del proceso de indagación (A-H) y las emociones manifestadas, siguiendo la taxonomía de Pekrun y Linnenbrink-García (2014), considerando como emociones positivas aquellas asociadas con el éxito y los desafíos (interés, satisfacción, concentración y confianza) y como negativas las relacionadas con el fracaso y el abandono (aburrimiento, insatisfacción, inseguridad, vergüenza y rechazo).

Tabla 4

Frecuencia de emociones por etapas y representación porcentual

\begin{tabular}{|c|c|c|c|c|c|c|c|c|c|c|c|}
\hline & \multirow[t]{2}{*}{ Emoción } & \multicolumn{8}{|c|}{$\begin{array}{c}\text { Frecuencia emociones } \\
\text { por etapas indagación }(*)\end{array}$} & \multirow{2}{*}{$\begin{array}{c}\text { TOTAL } \\
\text { (por emoción) }\end{array}$} & \multirow[t]{2}{*}{$\%$} \\
\hline & & $\mathbf{A}$ & B & $\mathrm{C}$ & D & $\mathbf{E}$ & $\mathrm{F}$ & G & $\mathbf{H}$ & & \\
\hline \multirow{5}{*}{ 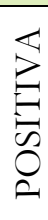 } & Interés & 6 & 3 & 2 & 5 & 3 & 5 & 6 & 5 & 35 & 41.2 \\
\hline & Confianza & 2 & 1 & 4 & 2 & 4 & 1 & 2 & 1 & 17 & 20.0 \\
\hline & Concentración & 1 & 4 & 3 & 3 & 2 & 3 & 1 & 0 & 17 & 20.0 \\
\hline & Satisfacción & 1 & 0 & 1 & 1 & 3 & 1 & 1 & 1 & 9 & 10.6 \\
\hline & Subtotal & 10 & 8 & 10 & 11 & 12 & 10 & 10 & 7 & 78 & 91.8 \\
\hline \multirow{3}{*}{$\underset{\substack{0 \\
Z}}{b}$} & Inseguridad & 0 & 3 & 1 & 0 & 0 & 0 & 0 & 1 & 5 & 5.9 \\
\hline & Insatisfacción & 0 & 0 & 0 & 1 & 0 & 1 & 0 & 0 & 2 & 2.4 \\
\hline & Aburrimiento & 0 & 0 & 0 & 0 & 0 & 0 & 0 & 0 & 0 & 0.0 \\
\hline
\end{tabular}




\begin{tabular}{ccccccccccc} 
Vergüenza & 0 & 0 & 0 & 0 & 0 & 0 & 0 & 0 & 0 & 0.0 \\
Rechazo & 0 & 0 & 0 & 0 & 0 & 0 & 0 & 0 & 0 & 0.0 \\
Subtotal & 0 & 3 & 1 & 1 & 0 & 1 & 0 & 1 & 7 & 8.2 \\
\hline $\begin{array}{c}\text { TOTAL } \\
\text { (por etapas) }\end{array}$ & 10 & 11 & 11 & 12 & 12 & 11 & 10 & 8 & 85 & 100.0 \\
\hline
\end{tabular}

(*) A: identificación de problemas investigables; B: formulación de hipótesis; C: identificación de variables; D: planificación de la investigación; E: recogida y procesamiento de datos; F: análisis de datos y obtención de conclusiones; G: comunicación de resultados; H: metarreflexión

De forma general, el $91.8 \%$ de las emociones seleccionadas por los PSFI tuvieron carácter positivo. Respecto a la tipología, «interés» fue la emoción positiva mayoritaria (41.2\%), seguida de «concentración» y «confianza», ambas con un 20\% de representación. Por el contrario, sólo dos emociones negativas fueron seleccionadas, «insatisfacción»e «inseguridad», ambas en bajo porcentaje (2.4 y 5.9, respectivamente). La representación de la frecuencia de emociones puede verse en la figura 2, en la que también se incluye el análisis porcentual por etapas.

\section{Figura 2}

Frecuencia de emociones positivas y negativas y porcentaje por etapas
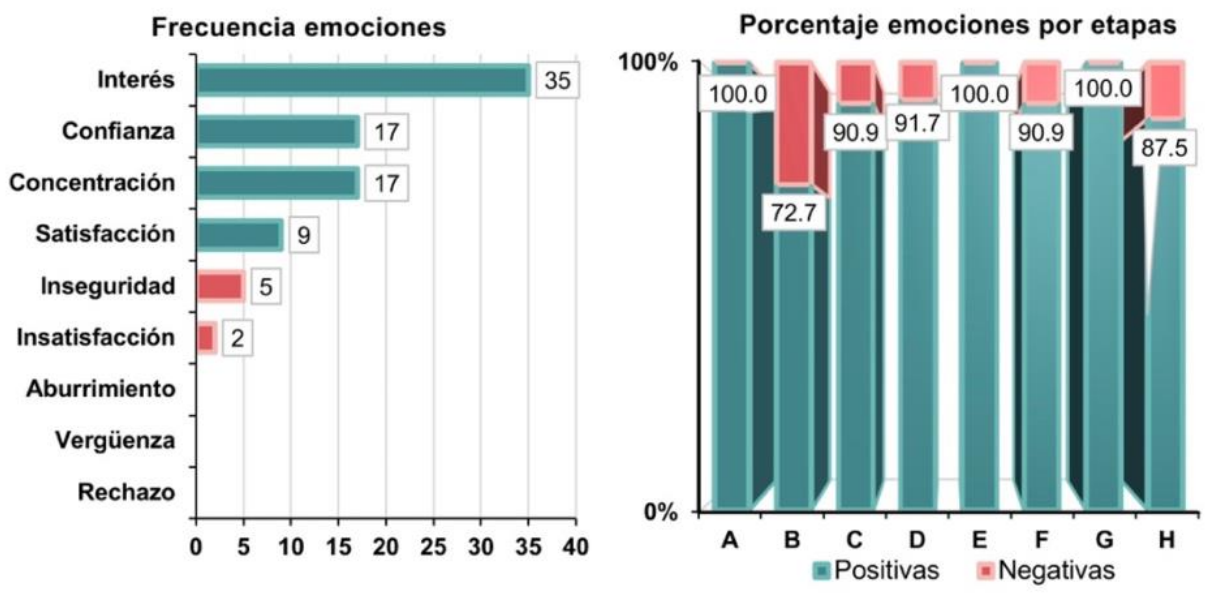

En cuanto a las etapas, destacan la identificación del problema investigable (A), la recogida y el procesamiento de datos (E) y la comunicación de resultados $(\mathrm{G})$, como etapas en las que no se asociaron emociones negativas. En las relacionadas con la identificación de variables $(\mathrm{C})$, la planificación de la investigación (D), el análisis de datos y obtención de conclusiones (F), y la metarreflexión (H), cerca del $90 \%$ de las emociones tuvo carácter positivo, y es la etapa referida a la formulación de hipótesis (B) la que asocia un menor porcentaje de emociones positivas $(72.7 \%)$. Estos valores pueden visualizarse de forma más concisa en la figura 2.

\subsection{Percepción sobre el aprendizaje}

La valoración otorgada por los PSFI sobre el aprendizaje adquirido antes y después de realizar la actividad de indagación, se recoge en la tabla 5 , donde aparecen las puntuaciones totales por participante y por etapa, registrando además la ganancia, expresada como la diferencia en el valor de la puntuación después y antes de realizar la actividad.

Tabla 5

Valoración de los PSFI (escala 1 a 5) sobre el aprendiraje adquirido acerca del proceso de indagación

\begin{tabular}{|c|c|c|c|c|c|c|c|c|c|c|c|}
\hline \multirow{2}{*}{ PSFI } & \multirow{2}{*}{ Tiempo } & \multicolumn{8}{|c|}{ Puntuación por etapas } & \multirow{2}{*}{ TOTAL } & \multirow{2}{*}{ GANANCIA } \\
\hline & & $\mathbf{A}$ & B & C & $\mathbf{D}$ & $\mathbf{E}$ & $\mathbf{F}$ & G & $\mathbf{H}$ & & \\
\hline 1 & $\begin{array}{l}\text { Antes de la actividad } \\
\text { Después de la actividad }\end{array}$ & $\begin{array}{l}3 \\
4\end{array}$ & $\begin{array}{l}3 \\
4\end{array}$ & $\begin{array}{l}3 \\
5\end{array}$ & $\begin{array}{l}3 \\
5\end{array}$ & $\begin{array}{l}3 \\
4\end{array}$ & $\begin{array}{l}3 \\
5\end{array}$ & $\begin{array}{l}3 \\
5\end{array}$ & $\begin{array}{l}2 \\
3\end{array}$ & $\begin{array}{l}23 \\
35\end{array}$ & +12 \\
\hline
\end{tabular}




\begin{tabular}{|c|c|c|c|c|c|c|c|c|c|c|c|}
\hline \multirow{2}{*}{2} & Antes de la actividad & 2 & 3 & 3 & 2 & 3 & 2 & 2 & 1 & 18 & \multirow[b]{2}{*}{+6} \\
\hline & Después de la actividad & 3 & 3 & 4 & 3 & 3 & 3 & 3 & 2 & 24 & \\
\hline \multirow[b]{2}{*}{3} & Antes de la actividad & 4 & 5 & 5 & 5 & 5 & 5 & 3 & 2 & 34 & \multirow[b]{2}{*}{+5} \\
\hline & Después de la actividad & 5 & 5 & 5 & 5 & 5 & 5 & 4 & 5 & 39 & \\
\hline \multirow{2}{*}{4} & Antes de la actividad & 4 & 4 & 3 & 2 & 4 & 4 & 4 & 1 & 26 & \multirow{2}{*}{+14} \\
\hline & Después de la actividad & 5 & 5 & 5 & 5 & 5 & 5 & 5 & 5 & 40 & \\
\hline \multirow{2}{*}{5} & Antes de la actividad & 2 & 3 & 4 & 3 & 4 & 4 & 3 & 1 & 24 & \multirow{2}{*}{+14} \\
\hline & Después de la actividad & 5 & 4 & 5 & 5 & 5 & 5 & 5 & 4 & 38 & \\
\hline \multirow[b]{2}{*}{6} & Antes de la actividad & 2 & 3 & 3 & 2 & 3 & 3 & 3 & 1 & 20 & \multirow{2}{*}{+7} \\
\hline & Después de la actividad & 3 & 3 & 4 & 3 & 4 & 4 & 4 & 2 & 27 & \\
\hline \multirow{2}{*}{7} & Antes de la actividad & 2 & 2 & 2 & 3 & 3 & 3 & 2 & 2 & 19 & \multirow{2}{*}{+7} \\
\hline & Después de la actividad & 3 & 3 & 3 & 3 & 4 & 4 & 3 & 3 & 26 & \\
\hline \multirow{5}{*}{8} & Antes de la actividad & 3 & 2 & 3 & 4 & 4 & 3 & 3 & 0 & 22 & \multirow{2}{*}{+11} \\
\hline & Después de la actividad & 5 & 4 & 5 & 5 & 5 & 5 & 4 & 0 & 33 & \\
\hline & TOTAL (antes) & 22 & 25 & 26 & 24 & 29 & 27 & 23 & 10 & & \\
\hline & TOTAL (después) & 33 & 31 & 36 & 34 & 35 & 36 & 33 & 24 & & \\
\hline & GANANCIA & +11 & +6 & +10 & +10 & +6 & +9 & +10 & +14 & & \\
\hline
\end{tabular}

La figura 3 representa gráficamente la diferencia en la percepción del aprendizaje por participante antes y después, y la ganancia adquirida por cada uno de ellos. En todos los casos, la valoración de los PSFI fue mayor al completar la actividad, con incremento que supera los diez puntos para los PSFI1, PSFI4, PSFI5 y PSFI8.

Figura 3

Valoraciones de los PSFI asociadas al aprendizaje adquirido antes y después de realizar la actividad de indagación
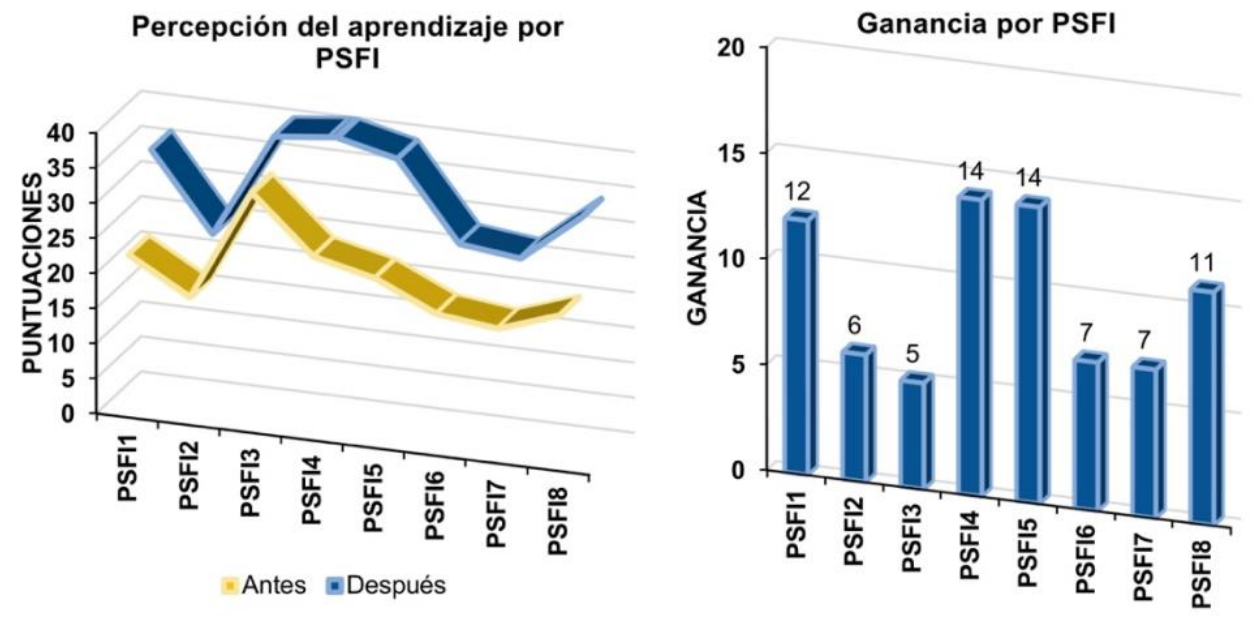

$\mathrm{Al}$ realizar el análisis por etapas (figura 4), se observa igualmente una mejora en la percepción después de la actividad, siendo en este caso la etapa de metarreflexión $(\mathrm{H})$ la que consigue una ganancia superior a los diez puntos, seguida por la etapa de identificación de problemas investigables (A), con once puntos. Por otro lado, son las etapas de formulación de hipótesis (B) y de recogida y procesamiento de datos (E) las que presentan menor ganancia, lo que podría indicar bien que los PSFI consideran que tienen un buen conocimiento de partida al respecto para ambas etapas o que, por el contrario, encuentran una dificultad adicional en su comprensión. 


\section{Figura 4}

Valoraciones del aprendizaje adquirido por etapas antes y después de realizar la actividad de indagación
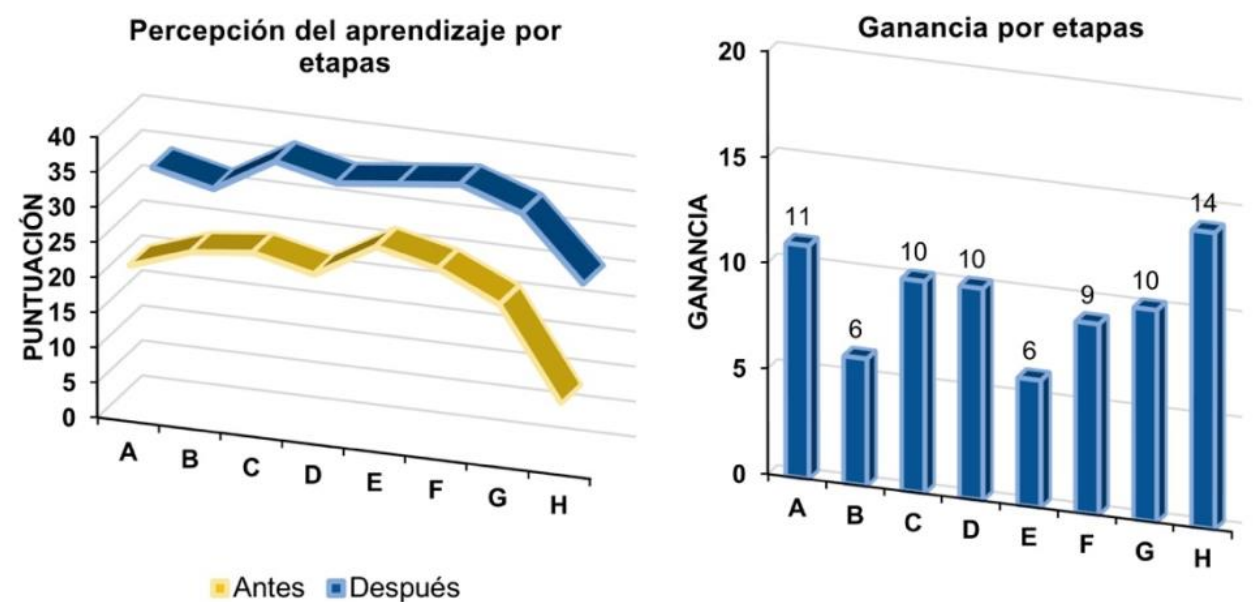

\subsection{Percepción de la aplicabilidad de la indagación}

El análisis cualitativo de las reflexiones de los PSFI se recoge en la tabla 6, donde se muestran las frecuencias de los 34 códigos asignados para las cuatro categorías principales (ventajas, inconvenientes, aplicación y modificaciones), asociadas a su vez con las preguntas planteadas en la actividad (P1-P4).

Tabla 6

Frecuencia de análisis de códigos

\begin{tabular}{|c|c|c|c|c|c|c|c|c|c|c|c|}
\hline \multirow[b]{2}{*}{ Pregunta } & \multirow[b]{2}{*}{ Categoría } & \multirow[b]{2}{*}{ Código } & \multicolumn{9}{|c|}{ Frecuencias códigos por PSFI } \\
\hline & & & 1 & 2 & 3 & 4 & 5 & 6 & 7 & 8 & TOTAL \\
\hline \multirow{15}{*}{ P1 } & \multirow{15}{*}{ Ventajas } & Actitudes científicas & 1 & 1 & 1 & & & & & & 3 \\
\hline & & Alfabetización científica & 1 & & & 1 & & & & & 2 \\
\hline & & Aprendizaje constructivista & & & & & 1 & & & 2 & 3 \\
\hline & & Aprendizaje significativo & & & & & & & & 1 & 1 \\
\hline & & Autonomía & 1 & 1 & 1 & & & & & & 3 \\
\hline & & Capacidad aroumentación & & 1 & 1 & & & & & & 2 \\
\hline & & Competencias científicas & & & & 1 & & & & 1 & 2 \\
\hline & & Contextualización & & & & & 1 & 1 & & 1 & 3 \\
\hline & & Multidisciplinariedad & & & & & & & & 1 & 1 \\
\hline & & Interés y motivación & 1 & 2 & 1 & 2 & 1 & 1 & 1 & 1 & 10 \\
\hline & & Desarrollo pensamiento crítico & 2 & 1 & 1 & & & & & & 4 \\
\hline & & Profundización contenidos & & & & & & & 1 & & 1 \\
\hline & & Toma de decisiones & & 1 & & & 1 & & & & 2 \\
\hline & & Trabajo cooperativo & 1 & 1 & & 1 & & & & 1 & 4 \\
\hline & & TOTAL & 7 & 8 & 5 & 5 & 4 & 2 & 2 & 8 & 41 \\
\hline \multirow{11}{*}{ P2 } & \multirow{11}{*}{ Inconvenientes } & Actitudes alumnado & 2 & & 1 & & & & & & 3 \\
\hline & & Gestión clase & & & 1 & & & & 1 & 1 & 3 \\
\hline & & Tiempo implementación & 1 & & & & 1 & 1 & & 1 & 4 \\
\hline & & Inexperiencia alumnado & & 1 & & & 1 & & & & 2 \\
\hline & & Inexperiencia docente & & 1 & & & & & & & 1 \\
\hline & & Implicación social & & 1 & & & & & & & 1 \\
\hline & & Grado guía & & & & & 2 & & & & 2 \\
\hline & & Individualización & & & & 1 & & & & & 1 \\
\hline & & Desarrollo profesional & & 1 & & & & & & & 1 \\
\hline & & Recursos & & & 1 & & & 1 & 1 & 1 & 4 \\
\hline & & Tiempo preparación & & 1 & & & & & & 1 & 2 \\
\hline
\end{tabular}




\begin{tabular}{|c|c|c|c|c|c|c|c|c|c|c|c|}
\hline & & Tipología alumnado & & & & & & 1 & & & 1 \\
\hline & & TOTAL & 3 & 5 & 3 & 1 & 4 & 3 & 2 & 4 & 25 \\
\hline \multirow{3}{*}{ P3 } & \multirow{3}{*}{ Aplicación } & Sí & 1 & 1 & 1 & 1 & 1 & 1 & 1 & 1 & 8 \\
\hline & & No & & & & & & & & & \\
\hline & & TOTAL & 1 & 1 & 1 & 1 & 1 & 1 & 1 & 1 & 8 \\
\hline \multirow{7}{*}{ P4 } & \multirow{7}{*}{ Modificación } & Adaptación previa & 1 & 1 & 1 & & & & 1 & & 4 \\
\hline & & Adaptación recursos & & & & & & 1 & & & 1 \\
\hline & & Aumento grado guía & & 1 & 1 & & 1 & & & 1 & 4 \\
\hline & & Aumento implicación alumnado & & 1 & & & & & & & 1 \\
\hline & & Coevaluación & 1 & & & & & & & & 1 \\
\hline & & Rotación roles cooperativos & & & & 1 & & & & 1 & 2 \\
\hline & & TOTAL & 2 & 3 & 2 & 1 & 1 & 1 & 1 & 2 & 13 \\
\hline
\end{tabular}

Entre las ventajas asociadas a la indagación, los PSFI manifestaron con mayor frecuencia «interés y motivación» (hasta diez menciones), seguido de «pensamiento crítico»y «trabajo cooperativo», ambas con cuatro menciones. Los principales inconvenientes encontrados fueron «tiempo de implementación» y «recursos», ambos con cuatro menciones.

$\mathrm{Si}$ bien todos los participantes coincidieron en que implementarían este tipo de actividades, igualmente expresaron la incorporación de alguna modificación, destacando en esta categoría la «adaptación previa» y el «aumento grado guía», con cuatro menciones respectivamente.

Adicionalmente, la búsqueda por palabra clave arrojó un recuento de seis menciones para «interés» por parte de cuatro de los PSFI, todas ellas en respuesta a la pregunta P1. En este apartado se consideraron además otras emociones no incluidas en los cuestionarios, siendo «miedo» la única citada por uno de los PSFI (PSFI2) en referencia a la pregunta P3.

\subsection{Identificación de perfiles de indagación}

Partiendo de que los ocho participantes afirmaron que aplicarían este tipo de actividad en su futura práctica docente, posteriormente, analizamos la relación entre las categorías con un mayor número de códigos asociados (ventajas e inconvenientes) (tabla 6), identificando tres perfiles de participantes bien diferenciados, y que se utilizaron como referencia en un análisis posterior individual: (i) denominado "altamente favorable a la indagación", en los casos que identificaban más del doble de ventajas que inconvenientes (PSFI1, PSFI4 y PSFI8), (ii) denominado "favorable a la indagación", cuando el número de ventajas frente a inconvenientes sigue siendo mayor (PSFI2 y PSFI3), pero sin alcanzar los valores del perfil (i), y (iii) denominado "ambivalente", cuando por el contrario, indicaban igual o mayor número de inconvenientes que ventajas (PSFI5, PSFI6 y PSFI7).

A continuación se describe cada uno de estos perfiles, analizando en ellos las posibles relaciones entre los tres aspectos investigados: emociones, percepción del aprendizaje y de la aplicabilidad de la indagación.

\section{PSFI altamente favorables a la indagación}

Dentro de este perfil se encuentran los PSFI1 (hombre), PSFI4 (mujer) y PSFI8 (mujer), siendo el perfil con mayor representación de mujeres. Como aspecto común, los tres participantes se encuentran en un rango de edad mayor de 30 años. Asimismo, estos participantes presentaron una alta ganancia en su percepción sobre el aprendizaje (tabla 5), superior a diez puntos en todos los casos. En la figura 5 se muestran los resultados de los tres participantes, que se analizarán de forma individual a continuación. 


\section{Figura 5}

Emociones y valoraciones de los PSFI altamente favorables a la indagación
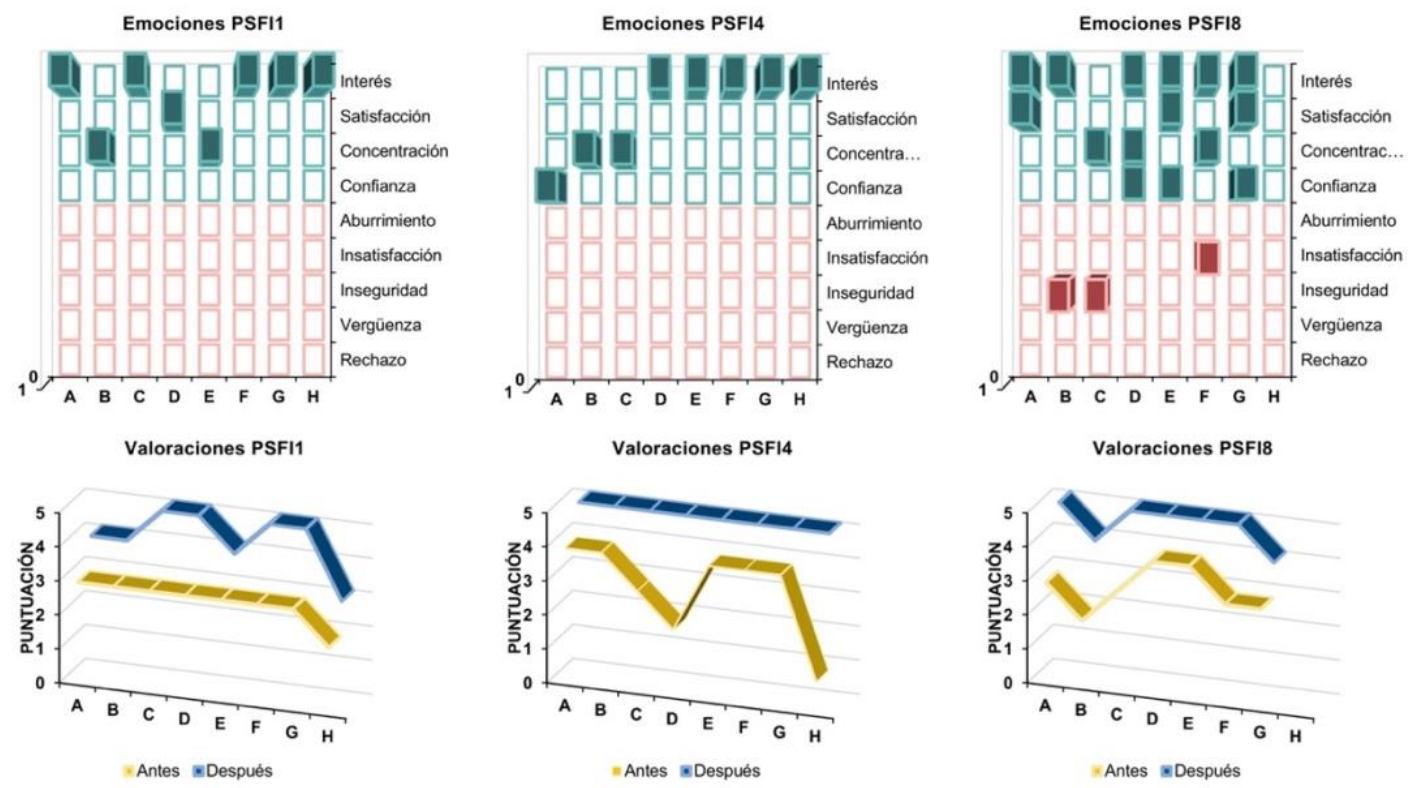

Para PSFI1, «interés» es la principal emoción positiva, y se correlaciona con el alto número de ventajas identificadas (7). Este participante experimentó también «concentración» en la formulación de hipótesis y la recogida y procesamiento de datos, aspectos que coinciden con una menor percepción de aprendizaje al respecto. En este caso, la etapa de metarreflexión es la que presenta menor valoración tanto inicial como final, indicando la necesidad de profundizar en este proceso durante la formación.

Igualmente, PSFI4 asoció «interés» a la mayor parte de las etapas, siendo notable los cambios en la percepción sobre el aprendizaje en torno a la planificación de la investigación y la metarreflexión. El perfil de partida de esta participante sobre la percepción del aprendizaje es bastante irregular, y sin embargo, otorga la máxima valoración a todas las etapas al completar la actividad, con un perfil final regular, lo que podría indicar una escasa reflexión del aprendizaje adquirido.

PSFI8 se encuentra entre los participantes que experimentaron un mayor número de emociones positivas durante el proceso, que se asocia con el elevado número de ventajas que describe (hasta 8) y es, además, la participante que más emociones negativas seleccionó. Concretamente, PSFI8 experimentó «inseguridad» al tratar la formulación de hipótesis y la identificación de variables, e «insatisfacción» en el análisis de datos y obtención de conclusiones). Una de estas etapas, la formulación de hipótesis, coincide con la menor puntuación sobre su percepción del aprendizaje. Esta «inseguridad», que manifestó tras realizar la indagación, podría relacionarse con el aumento del grado de guía en la experiencia, que propone como modificación («Creo que uno de los principales problemas que surgen es el número de variables que se establecen, por ello, sería interesante que el docente limitara el número de variables a modificar a la hora de la experimentación, y así facilitar la obtención de conclusiones»). Esta participante no ha valorado ni puntuado la etapa de metarreflexión, bien porque no considera haberla realizado, o porque no ha sido consciente de ello, lo que refuerza la idea de trabajar en este proceso con mayor énfasis.

\section{PSFI favorables a la indagación}

En este perfil, en el que todavía se identifica un mayor número de ventajas que de inconvenientes asociados a la indagación, encajan dos de los tres participantes PSFI2 (hombre) y PSFI3 (mujer) que seleccionaron emociones negativas en algún momento del proceso, mostrándose sus resultados en la figura 6. PSFI3 es la única de los ocho que tiene una experiencia en investigación, tras haber realizado el doctorado en física. Ambos participantes registraron la menor ganancia sobre la percepción del aprendizaje (tabla 5), con una puntuación igual of inferior a seis en ambos casos. 


\section{Figura 6}

Emociones y valoraciones de los PSFI favorables a la indagación
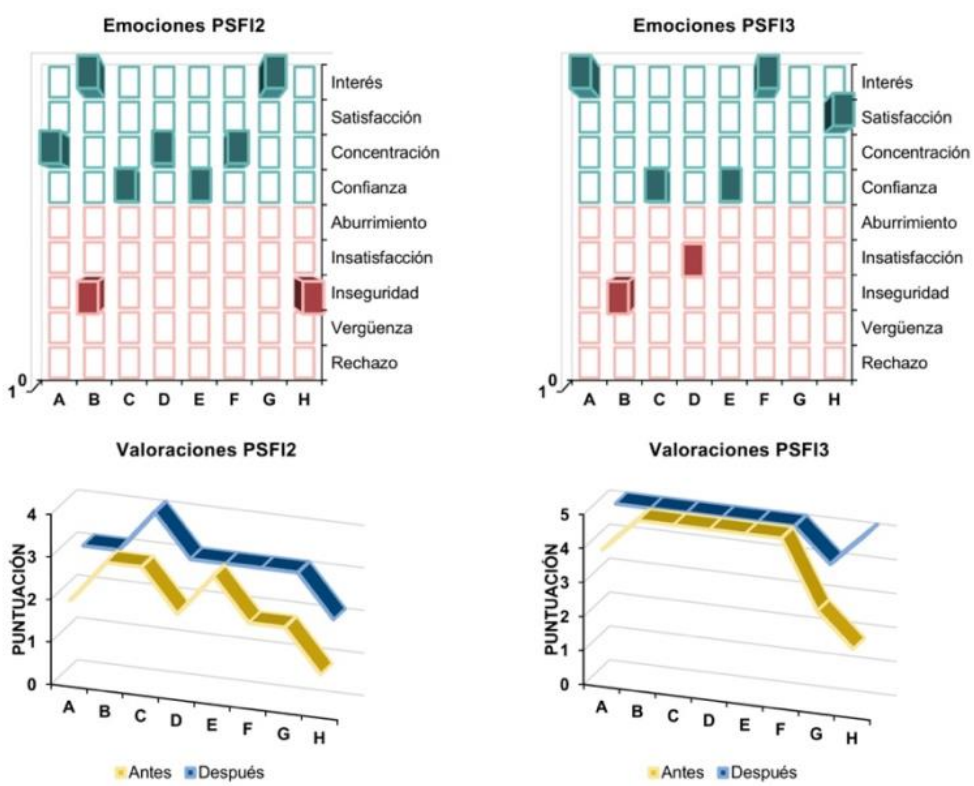

Para PSFI2, la «inseguridad» está asociada a dos procesos: la formulación de hipótesis y la metareflexión, y coinciden con su menor percepción de aprendizaje al respecto. Aunque este participante experimenta igualmente emociones positivas en las restantes etapas («interés» para B y G, «concentración» para A, D y F, y «confianza» para $\mathrm{C}$ y E), el peso de las emociones negativas se reflejó en la búsqueda por palabra clave realizada en las reflexiones, en la que emergió «miedo» como emoción asociada a la implementación de propuestas de indagación («debo reconocer que me da un poco de miedo el que, por mi falta de experiencia, no tenga toda la utilidad para el alumno que se pretende»).

En el caso de PSFI3, igualmente la formulación de hipótesis llevó asociada «inseguridad». Además, en la planificación de la investigación, esta participante experimentó «insatisfacción», aspecto que puede estar relacionado con los principales inconvenientes encontrados, «actitudes del alumnado» y «gestión de clase» («requiere de una mayor organización y control del grupo-clase por parte del docente») y con las modificaciones propuestas (adaptación previa del alumnado a la metodología y aumento del grado de guía por parte del docente). Fruto de la «insatisfacción» derivada de la planificación, PSFI3 expone que «desde mi perspectiva no dejaría que los alumnos cambiasen más de una variable a la vez, se desvirtúa el método científico». Esta afirmación podría indicar una diferenciación entre su perfil investigador (que podría estar bastante arraigado como consecuencia de su etapa de doctorado) y su futuro perfil docente, ya que, si bien es consciente de la importancia de una buena planificación de la investigación y de la importancia en la selección y tipología de las variables, no parece contemplar la necesidad de reflexionar con el alumnado sobre el proceso de selección y control de variables y ayudarles a que sean ellos los que plantean las consecuencias de modificar más de una variable en cada experimento. Fue la participante que menor ganancia sobre el aprendizaje mostraba $(+5)$ (tabla 5), lo que puede ser debido a que ya conocía suficientemente las etapas de la indagación por su formación previa como investigadora. No obstante, en la etapa que más evolucionó fue en la de metarreflexión, quizás por tratarse de un aspecto novedoso para ella.

\section{PSFI ambivalentes a la indagación}

Entre los PSFI que identificaron igual o mayor número de inconvenientes que de ventajas en la aplicación de la indagación se encuentran los tres últimos participantes, PSFI5, PSFI6 y PSFI7, que presentan como rango común el género masculino. En este grupo se encuentran dos de los participantes que no habían tenido experiencia docente previa (PSFI5 y PSFI6) y el único de la muestra con una experiencia docente universitaria (PSFI7). Todos ellos seleccionaron exclusivamente emociones de carácter positivo durante el proceso.

En este grupo encontramos un hecho diferenciador en cuanto a la percepción del aprendizaje. Mientras que PSFI6 y PSFI7 presentaron una ganancia de siete puntos (tabla 5), PSFI5 presentó una ganancia de 
catorce, encontrándose este resultado más en consonancia con los PSFI1, PSFI4 y PSFI8, pertenecientes al perfil de participantes altamente favorable. En la figura 7 se muestran los resultados de emociones y valoraciones sobre el aprendizaje otorgadas por cada uno de ellos.

\section{Figura 7}

Emociones y valoraciones de los PSFI ambivalentes
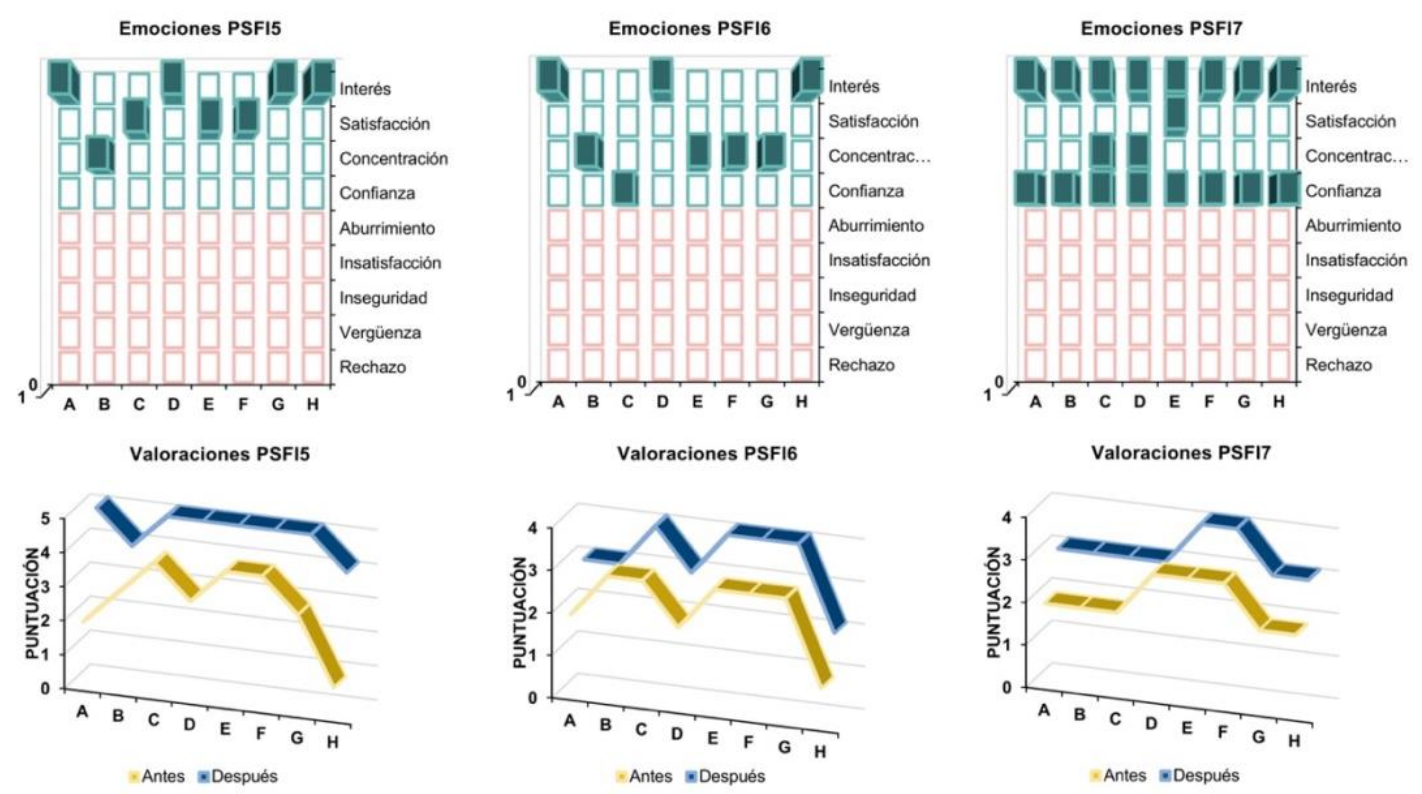

Así, para PSFI5 todo el proceso de indagación estuvo asociado a emociones positivas, resaltando «interés» para las etapas A, D, G y H, en las que considera haber incorporado un mayor aprendizaje, y «satisfacción» para las etapas C, E y F. Un aspecto que destacar es la elección de «concentración» durante la formulación de hipótesis, una etapa con escasa mejora $(+1)$, lo que puede entenderse como una posible dificultad en esta etapa. Por otro lado, el principal inconveniente aportado en su reflexión, mencionado en dos ocasiones, se refiere al grado de guía del docente durante la indagación («la falta de costumbre del alumnado ante este tipo de prácticas puede suponer una desventaja a la hora de implementar la actividad, ya que requerirá un mayor control del profesor»), y que contempla igualmente como sugerencia de modificación de cara a una posible puesta en práctica («aunque los alumnos decidan qué variables quieren estudiar, creo que debe hacerse con un poco más de rigor»).

Un perfil similar se describe para PSFI6, siendo esta vez «concentración» la emoción mayoritaria, asociada a las etapas B, E, F y G, coincidiendo además el proceso de formulación de hipótesis como la única etapa en la que no se manifestó mejora en su aprendizaje. PSFI6 no identifica ninguna ventaja asociada al aprendizaje (tabla 6) y los inconvenientes que relaciona están referidos a aspectos contextuales (tiempo de implementación y recursos), en línea de lo que se ha constado en otros trabajos (Abril et al., 2014; RomeroAriza et al., 2020) o a la tipología de alumnado.

Por último, PSFI7 es el participante que mayor número de emociones positivas muestra, entre las que selecciona «interés» y «confianza» para todas las etapas. Llama la atención que, además de «interés y motivación», PSFI7 solo aportara "profundización contenidos» como ventaja de la indagación. Aunque existen referencias sobre cómo este enfoque puede contribuir, discretamente, a una mejora en el conocimiento de los contenidos (Romero-Ariza, 2017), no es quizás una de sus potencialidades, habiendo pasado desapercibidas las restantes para PSFI7. Quizás su amplia experiencia docente en la educación superior universitaria (en grados de ingeniería) le ha llevado a centrarse en este aspecto.

Para concluir, y con idea de facilitar una visión global de los resultados obtenidos, la tabla 7 recoge, a modo de resumen, las relaciones entre las emociones y la percepción del aprendizaje manifestado por los PSFI de cada uno de los perfiles analizados. 
Tabla 7

Relaciones entre percepción de aplicación de la indagación, emociones y percepción de aprendizaje

\begin{tabular}{ccccccc}
\hline \multirow{2}{*}{ PERFIL } & \multirow{2}{*}{ PSFI } & \multirow{2}{*}{ GÉNERO } & \multicolumn{2}{c}{ EMOCIONES } & \multirow{2}{*}{ APRENDIZAJE } \\
\cline { 4 - 6 } & & & POSITIVAS & NEGATIVAS & \\
Altamente & 1 & $\mathrm{H}$ & 8 & 0 & +12 \\
favorable & 4 & $\mathrm{M}$ & 8 & 0 & +14 \\
& 8 & $\mathrm{M}$ & 15 & 3 & +11 \\
\hline \multirow{2}{*}{ Favorable } & 2 & $\mathrm{H}$ & 7 & 2 & +6 \\
& 3 & $\mathrm{M}$ & 5 & 2 & +5 \\
\hline \multirow{2}{*}{ Ambivalente } & 5 & $\mathrm{H}$ & 8 & 0 & +14 \\
& 6 & $\mathrm{H}$ & 8 & 0 & +7 \\
& 7 & $\mathrm{H}$ & 19 & 0 & +7 \\
\hline
\end{tabular}

\section{Discusión y conclusiones}

Se plantean, a continuación, la discusión de los resultados y las conclusiones organizadas en torno a las preguntas de investigación. Finalmente, se discuten algunas limitaciones del estudio e implicaciones para la investigación y la formación inicial del profesorado.

¿Qué emociones manifiestan profesores de Física y Química en formación inicial cuando llevan a cabo una actividad de indagación?

Las emociones experimentadas por los PSFI tras la realización de una actividad de indagación que sirve de introducción al conocimiento didáctico del contenido de este enfoque (García-Ruiz et al., 2020) son altamente favorecedoras, con la totalidad de los participantes experimentando emociones positivas, principalmente de carácter epistémicas («interés» y «concentración»). Estos resultados son congruentes con los reportados en la bibliografía, al identificar los PSFI un gran «interés» en el proceso de indagación, sin duda consecuencia de la inclusión de una actividad de indagación como parte de su formación inicial (Alvarado et al., 2019; Jiménez-Liso et al., 2019).

En el grupo de las emociones negativas se han obtenido resultados similares a los descritos por SánchezMartin et al. (2018), asociados a etapas concretas de la indagación, si bien en nuestro estudio ningún participante manifestó «rechazo», y sí «inseguridad» e «insatisfacción». Dentro de este grupo se identifica la principal emoción negativa manifestada («inseguridad»), seleccionada por tres de los 8 PSFI. Del análisis de los perfiles individuales se ha podido comprobar que esta emoción se encuentra íntimamente ligada a la etapa de formulación de hipótesis, en la que, además, se observa una percepción del aprendizaje menor. Esta falta de seguridad se manifiesta en la necesidad de realizar las actividades de indagación con un mayor nivel de guía que con el que ellos habían llevado a cabo la actividad. En estos momentos del programa formativo parece lógico encontrar cierta "inseguridad", que pueden estar relacionada con una falta de confianza en sus habilidades docentes y que, como indican diversos estudios (Krämer et al., 2015; Nuangchalerm, 2017; Qablan, 2008) se traduce en adoptar indagaciones con un alto grado de guía por parte del profesor. Por ello, es necesario abordar de forma explícita en los programas formativos, como se hizo en el del presente estudio (García-Ruiz et al., 2020), la cuestión del "grado de apertura" de las actividades de indagación y de cómo los profesores pueden ir ajustándolo en función de las características de sus estudiantes, en particular de su grado de familiaridad con este tipo de actividades.

Considerando el efecto activador que las emociones epistémicas positivas tienen en el logro de las expectativas docentes (Mellado et al., 2014), encontramos satisfactorios y esperanzadores las emociones manifestadas por los PSFI en este estudio, que perciben la indagación como una práctica de interés. Ahora bien, la ausencia de menciones emocionales en las reflexiones de los participantes, con sólo una expresión generalizada de «interés» y una mención individual a «miedo» no hace sino poner en evidencia la dificultad que encuentran a la hora de expresar emociones que, a priori, no se manifiestan espontáneamente, lo que requiere de un proceso de explicitación que favorezca su identificación y reconocimiento.

¿Qué percepciones tienen sobre su propio aprendizaje después de realizar la actividad de indagación?

Los resultados obtenidos muestran que todos los PSFI consideran que involucrarse en la actividad de indagación les ha ayudado a mejorar su aprendizaje sobre las distintas etapas que la constituyen. No obstante, se aprecia una amplia dispersión en el grado de dicha mejora, desde +5 puntos hasta +14 puntos. Aunque no se aprecia una relación entre el grado de mejora y otras características de los participantes (género, edad, titulación académica, experiencia investigadora o docente), sí se puede resaltar que dos de los participantes que mostraban menores cambios en la percepción de su aprendizaje eran los que poseían una mayor 
experiencia investigadora (PSFI3) o experiencia docente en el ámbito universitario (PSFI7). Estos resultados parecen lógicos, si se considera que quizás los participantes no hayan tenido oportunidad de realizar actividades de indagación durante sus etapas de estudiantes, teniendo en cuenta que este enfoque no está todavía muy extendido en la práctica educativa (Vázquez y Manassero, 2015).

Con respecto a las etapas de la indagación, observamos una menor evolución en las relacionadas con la formulación de hipótesis o la recogida y el procesamiento de datos, siendo estos aspectos en los que hay que profundizar más durante la formación. En contraposición, el cambio más llamativo se refiere a los procesos de metarreflexión y de identificación de problemas investigables, lo que podría indicar un desconocimiento inicial al respecto.

¿Qué percepción tienen, después de realizar la actividad, sobre la aplicabilidad de la indagación en las clases de Física y Química?

Todos los PSFI participantes afirmaron que aplicarían la indagación en sus futuras clases de ciencias, lo que podría indicar que todos están igualmente predispuestos a ello. El análisis cualitativo de sus reflexiones ha permitido identificar un buen número de ventajas y algunos inconvenientes expresados por los PSFI, en la línea de lo planteado en estudios anteriores sobre las bondades de la indagación (Chen et al., 2014; Marshall et al., 2016) o sobre dificultades para su implementación (Abril et al., 2014; Romero-Ariza et al., 2020). El balance entre el número de ventajas e inconvenientes indicados por cada PSFI ha sido utilizado como criterio para identificar perfiles más o menos favorables a la indagación. En función de los cuáles se puede concluir que 5 de los 8 participantes pueden considerarse favorables a la indagación, de ellos 3 muy favorables, y 2 favorables. En nuestro estudio, además, hemos observado una diferencia de género, con una mayor representación de las mujeres en los perfiles más favorables a la indagación (tabla 7).

¿Qué relaciones, si las hay, aparecen entre las emociones manifestadas, la percepción de su aprendizaje y la aplicabilidad de la indagación?

En este trabajo no se encuentra relación entre el número de emociones positivas experimentadas y la aplicabilidad de la indagación en el aula, con PSFI altamente favorables a la indagación que todavía experimentan alguna emoción negativa, y PSFI ambivalentes a la indagación cuya experiencia durante el proceso de indagación fue exclusivamente positiva. En aquellos casos en los que se expresó una emoción negativa, los PSFI continuaron identificando ventajas, pudiendo traducirse en una escasa influencia de las emociones negativas de cara a la futura implementación de la indagación, lo que parece no estar de acuerdo con lo planteado por diversos autores (Tomas et al., 2016). Sí se aprecia, en cambio, una cierta relación entre los perfiles de aplicabilidad y la percepción sobre el aprendizaje. Los tres estudiantes del perfil altamente favorable a la indagación indicaban que habían aprendido bastante sobre indagación, mientras que, en los perfiles favorables y ambivalentes, la percepción sobre su aprendizaje es notablemente menor, excepto en el caso del PSF5.

Los resultados y las conclusiones que se plantean se han obtenido con un número reducido de PSFI, lo que nos lleva a considerar que no podemos establecer conclusiones generalizables, y a entender esta investigación como un estudio exploratorio que ha hecho emerger algunas hipótesis de trabajo de cara a investigaciones futuras. En este sentido, la percepción de los PSFI sobre el aprendizaje acerca de la indagación y el grado de aplicabilidad en el aula son dos aspectos que parecen estar relacionados. De ser así, parece necesario que los PSFI, si no lo han hecho en sus etapas de estudiantes, se involucren en actividades de indagación y reflexionen sobre su propio aprendizaje durante ellas. Si estas actividades les ayudan a aprender, puede que los PSFI asuman que también pueden ayudar a aprender a sus futuros estudiantes.

Finalmente, considerando el efecto directo que presenta para la formación inicial el diseño de actividades que enfaticen la participación activa y la construcción del conocimiento, encontramos que la estrategia diseñada ha contribuido a promover el interés y las emociones positivas, haciendo partícipe a los PSFI de los procesos de indagación y proporcionándole una forma de autoconocimiento al respecto. No obstante, encontramos necesario continuar trabajando en esta línea, con futuros estudios que proporcionen herramientas para una mejor autorregulación emocional.

\section{Apoyos}

Este estudio forma parte del proyecto de I+D de Excelencia "Desarrollo de competencias en problemas de la vida diaria mediante prácticas científicas de argumentación, indagación y modelización en enseñanza secundaria y universitaria (CPAIM)" (EDU2017-82197-P) financiado por el Ministerio de Ciencia, Innovación y Universidades 2017. La Dra. García-Ruiz agradece al Plan Propio de la Universidad de Málaga por la "Ayuda para la incorporación de doctores" recibida. 


\section{Referencias}

Abril, A. M., Ariza, M. R., Quesada, A. y García, F. J. (2014). Creencias del profesorado en ejercicio y en formación sobre el aprendizaje por investigación. Revista Eureka sobre Enseñanza y Divulgación de las Ciencias, 11(1), 22-33. https://doi.org/10498/15710

Alvarado, D. A. R., De las Heras Pérez, M. Á., Vázquez-Bernal, B. y Jiménez-Pérez, R. (2019). ¿Cómo cambian las emociones en docentes en formación inicial al participar en un proyecto de indagación en el aula? Apice. Revista de Educación Cientifica, 3(2), 55-69. https://doi.org/10.17979/arec.2019.3.2.4629

Bartley, J. (2019). A longitudinal study of the progressioin of preservice and in-service science teachers' abilities to teach inquiry-based science. Illinois Institute of Technology.

Bellocchi, A., Quigley, C. y Otrel-Cass, K. (Eds.) (2017). Exploring emotions, aesthetics and wellbeing in science education research. Springer International Publishing.

Bryman, A. (2006). Integrating quantitative and qualitative research: how is it done? Qualitative Research, 6(1), 97-113. https://doi.org/10.1177/1468794106058877

Chen, H. T., Wang, H. H., Lin, H. S., Lawrenz, F. P. y Hong, Z. R. (2014). Longitudinal study of an afterschool, inquiry-based science intervention on low-achieving children's affective perceptions of learning science. International Journal of Science Education, 36(13), 2133-2156. https://doi.org/10.1080/09500693.2014.910630

Chiu, M. H., Chou, C. C., Wu, W. L. y Liaw, H. (2014). The role of facial microexpression state (FMES) change in the process of conceptual conflict. British Journal of Educational Technology, 45(3), 471-486. https://doi.org/10.1111/bjet.12126

Constantinou, C. P., Tsivitanidou, O. E. y Rybska, E. (2018). What is inquiry-based science teaching and learning? En O. E. Tsivitanidou, P. Gray, E. Rybska, L. Louca, y C. P. Constantinou (Eds.), Professional Development for Inquiry-Based Science Teaching and Learning (Vol. 5, pp. 1-26). Springer.

Crawford, B. A. (2014). From inquiry to scientific practices in the science classroom. En N. G. Lederman y S. K. Abell (Eds.), Handbook of Research on Science Education. Routledge. https://doi.org/10.4324/9780203097267

D’Mello, S. y Graesser, A. (2012). Dynamics of affective states during complex learning. Learning and Instruction, 22(2), 145-157. https://doi.org/10.1016/j.learninstruc.2011.10.001

Davis, J. P. y Bellocchi, A. (2018). Objectivity, subjectivity, and emotion in school science inquiry. Journal of Research in Science Teaching, 55(10), 1419-1447. https://doi.org/10.1002/tea.21461

Ferrés-Gurt, C., Marbà-Tallada, A. y Sanmartí-Puig, N. (2015). Trabajos de indagación de los alumnos: instrumentos de evaluación e identificación de dificultades. Revista Eureka sobre Enseñanza y Divulgación de las Ciencias, 12(1), 22-37.

García-Ruiz, C., Lupión-Cobos, T. y Blanco-López, A. (2020). Propuesta formativa sobre indagación para profesorado de física y química en formación inicial. En D. Cebrián-Robles, A. J. Franco-Mariscal, T. Lupión-Cobos, C. Acebal-Expósito y A. Blanco-López (Coords.), Enseñanza de las ciencias y problemas relevantes de la ciudadanía. Graó (en prensa).

Jiménez-Liso, M. R., Martínez-Chico, M., Avraamidou, L. y López-Gay, R. (2019). Scientific practices in teacher education: the interplay of sense, sensors, and emotions. Research in Science and Technological Education, 1-24. https://doi.org/10.1080/02635143.2019.1647158

Kang, E. J. S., Bianchini, J. A. y Kelly, G. J. (2013). Crossing the border from science student to science teacher: preservice teachers' views and experiences learning to teach inquiry. Journal of Science Teacher Education, 24(3), 427-447. https://doi.org/10.1007/s10972-012-9317-9

Krämer, P., Nessler, S. H. y Schlüter, K. (2015). Teacher students' dilemmas when teaching science through inquiry. Research in Science and Technological Education, 33(3), 325-343. https://doi.org/10.1080/02635143.2015.1047446

Marshall, J. C., Smart, J. B. y Alston, D. M. (2016). Inquiry-based instruction: a possible solution to improving student learning of both science concepts and scientific practices. International Journal of Science and Mathematics Education, 15, 777-796. https://doi.org/10.1007/s10763-016-9718-x

McLaughlin, C. A. y MacFadden, B. J. (2014). At the elbows of scientists: shaping science teachers' conceptions and enactment of inquiry-based instruction. Research in Science Education, 44(6), 927-947. https://doi.org/10.1007/s11165-014-9408-z

Mellado, V., Borrachero, A. B., Brígido, M., Melo, L. V., Dávila, M. A., Cañada, F., Conde, M. C., Costillo, E., Cubero, J., Esteban, R., Martínez, G., Ruiz, C., Sánchez, J., Garritz, A., Mellado, L., Vázquez, B., Jiménez, R. y Bermejo, M. L. (2014). Las emociones en la enseñanza de las ciencias. Enseñanza de las Ciencias, 32(3), 11-36. https://doi.org/10.5565/rev/ensciencias.1478 
Muñoz-Campos, V., Franco-Mariscal, A. J. y Blanco-López, A. (2020). Integración de prácticas científicas de argumentación, indagación y modelización en un contexto de la vida diaria. Valoraciones de estudiantes de secundaria. Revista Eureka sobre Enseñanza y Divulgación de las Ciencias, 17(3), 3201. https://doi.org/10.25267/Rev_Eureka_ensen_divulg_cienc.2020.v17.i3.3201

Nuangchalerm, P. (2017). Relationship between preferred and actual opinions about inquiry-based instruction classsroom. European Journal of Science and Mathematics Education, 5(1), 67-73.

Organisation for Economic Co-operation and Development [OECD]. (2017). PIS A 2015 Assessment and analytical framework. Science, reading, mathematic, financial literacy and collaborative problem solving (revised edition). OECD Publishing. https://doi.org/https://doi.org/10.1787/9789264281820-en

Palmer, D. H. (2009). Student interest generated during an inquiry skills lesson. Journal of Research in Science Teaching, 46(2), 147-165. https://doi.org/10.1002/tea.20263

Pekrun, R. y Linnenbrink-García, L. (Eds.) (2014). International Handbook of Emotions in Education. Routledge. https://doi.org/10.4324/9780203148211.ch3

Pekrun, R., Muis, K. R., Frenzel, A. C., y Goetz, T. (2018). Emotions at school (Vol. 17). Routledge.

Qablan, A. (2008). Why are we doing this? Preservice science teachers' interpretations of the role of open inquiry in understanding teaching. Journal of Institutional Research, 14(1), 24-42.

Ritchie, S. M., Tobin, K., Sandhu, M., Sandhu, S., Henderson, S. y Roth, W. M. (2013). Emotional arousal of beginning physics teachers during extended experimental investigations. Journal of Research in Science Teaching, 50(2), 137-161. https://doi.org/10.1002/tea.21060

Rocard, M., Csermely, P., Jorde, D., Walberg-Henriksson, H. y Hemmo, V. (2007). Science Education now: a renewed pedagogy for the future of Europe. European Communities.

Romero-Ariza, M. (2017). El aprendizaje por indagación: ¿existen suficientes evidencias sobre sus beneficios en la enseñanza de las ciencias? Revista Eureka sobre Enseñanza y Divulgación de las Ciencias, 14(2), 286299. https://doi.org/10498/16933

Romero-Ariza, M., Quesada, A., Abril, A. M., Sorensen, P. y Oliver, M. C. (2020). Highly recommended and poorly used: English and Spanish science teachers' views of inquiry-based learning (IBL) and its enactment. Eurasia Journal of Mathematics, Science and Technology Education, 16(1), 1-16.

Saldaña, J. (2009). The coding manual for qualitative researchers. SAGE.

Sanchez-Martin, J., Cañada-Cañada, F. y Dávila-Acedo, M. A. (2018). Emotional responses to innovative science teaching methods: acquiring emotional data in a general science teacher education class. Journal of Technology and Science Education, 8(4), 346-359. https://doi.org/10.3926/jotse.408

Sjøberg, S. (2019). Critical perspectives on inquiry-based science education (IBSE) in Europe. Position Paper Written for EUN Partnership, European Schoolnet, March.

Tomas, L., Rigano, D.,y Ritchie, S. M. (2016). Students' regulation of their emotions in a science classroom. Journal of Research in Science Teaching, 53(2), 234-260. https://doi.org/10.1002/tea.21304

Vázquez, A., y Manassero, M. A. (2015). Hacia una formación inicial del profesorado de ciencias basada en la investigación. Revista Española de Pedagogía, 261, 343-363.

Yeigh, T., Woolcott, G., Scott, A., Donnelly, J., Snow, M. y Whannell, R. (2016). Emotional literacy and pedagogical confidence in pre-service science and mathematics teachers. Australian Journal of Teacher Education, 41(6), 107-121. https://doi.org/10.14221/ajte.2016v41n6.7

Zion, M., Schwartz, R. S., Rimerman-Shmueli, E.,y Adler, I. (2018). Supporting teachers' understanding of nature of science and inquiry through personal experience and perception of inquiry as a dynamic process. Research in Science Education, 50, 1281-1304. https://doi.org/10.1007/s11165-018-9732-9 